\title{
Early tholeiitic and calc-alkaline arc magmatism of middle to Late Eocene Age in the southern Ogasawara (Bonin) forearc
}

\author{
Satoru Haraguchi · Teruaki Ishii · Jun-Ichi Kimura
}

Received: 25 March 2008/Accepted: 7 April 2008/Published online: 3 June 2008

(c) Springer-Verlag 2008

Erratum to: Contrib Mineral Petrol (2008) 155:593-618 DOI 10.1007/s00410-007-0260-2

In Fig. 2 at line 3 from the bottom in the legend, the solid square symbol and the word "this study" should be deleted. Please find the corrected figure below.
In Table 3 on the bottom line, both of the $\mathrm{K}-\mathrm{Ar}$ dates are incorrectly positioned. The date of $39.4 \pm 0.2$ should be positioned in the column D13-05. The date of $45.0 \pm 1.8$ should be positioned in the column D16-101. Please find the corrected table below.

The online version of the original article can be found under doi:10.1007/s00410-007-0260-2.

S. Haraguchi $(\bowtie) \cdot$ T. Ishii

Ocean Research Institute, University of Tokyo,

Nakano 164-8639, Japan

e-mail: haraguti@ori.u-tokyo.ac.jp

Present Address:

T. Ishii

Institute for Frontier Research Science and Technology (IFREE),

Japan Agency for Marine-Earth Science and Technology

(JAMSTEC), Yokosuka 237-0061, Japan

J.-I. Kimura

Department of Geoscience, Shimane University,

Matsue 690-8504, Japan 
Fig. 2 Space-time distribution of volcanic activity on the Philippine Sea plate (modified from Haraguchi et al. 2003), including $\mathrm{K}-\mathrm{Ar}$ (Kaneoka et al. 1970; Tsunakawa 1983) and Ar-Ar ages (Cosca et al. 1998; Dobson 1986; Ishizuka et al. 2006) of Chichi-Jima boninite (solid circles) and Haha-Jima volcanics (open circles)

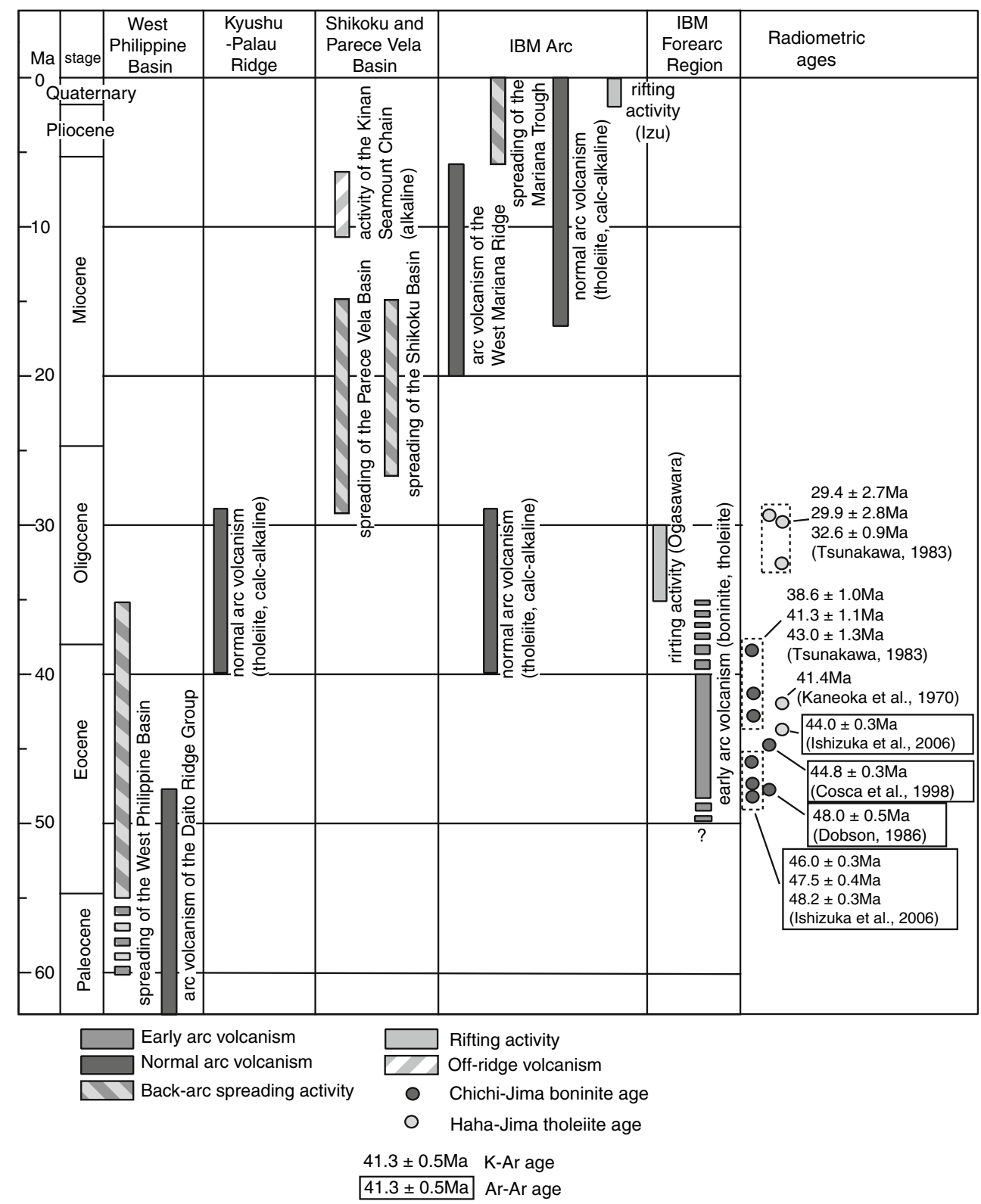

Table 3 Bulk rock composition of volcanic rocks estimated by ICP-MS from the southern Ogasawara Ridge dredged during cruises KT95-9

\begin{tabular}{|c|c|c|c|c|c|c|c|c|c|c|c|c|}
\hline \multirow{2}{*}{$\begin{array}{l}\text { Sample } \\
\text { ID }\end{array}$} & \multicolumn{6}{|c|}{ KT95-9 D13 } & \multirow{2}{*}{$\begin{array}{l}\text { D14 } \\
\text { D14-05 }\end{array}$} & \multicolumn{5}{|l|}{ D16 } \\
\hline & D13-04 & D13-05 & D13-14 & D13-24 & D13-25 & D13-112 & & D16-101 & D16-103 & D16-105 & D16-109 & D16-202 \\
\hline \multicolumn{13}{|c|}{ Major elements (wt\%) } \\
\hline $\mathrm{SiO}_{2}$ & 52.43 & 53.21 & 49.46 & 52.38 & 54.39 & 54.43 & 55.40 & 53.08 & 55.85 & 55.42 & 53.67 & 57.88 \\
\hline $\mathrm{TiO}_{2}$ & 0.94 & 0.85 & 0.77 & 0.82 & 1.02 & 1.01 & 0.58 & 0.49 & 0.45 & 0.65 & 0.48 & 0.52 \\
\hline $\mathrm{Al}_{2} \mathrm{O}_{3}$ & 18.29 & 17.78 & 18.42 & 18.25 & 17.82 & 17.28 & 19.07 & 18.44 & 18.55 & 17.20 & 18.43 & 19.39 \\
\hline $\mathrm{Fe}_{2} \mathrm{O}_{3}$ & 9.99 & 9.62 & 10.44 & 9.15 & 9.85 & 10.13 & 6.41 & 8.07 & 7.75 & 8.00 & 8.27 & 6.11 \\
\hline $\mathrm{MnO}$ & 0.15 & 0.15 & 0.15 & 0.13 & 0.15 & 0.15 & 0.13 & 0.13 & 0.14 & 0.14 & 0.14 & 0.08 \\
\hline $\mathrm{MgO}$ & 4.90 & 4.96 & 5.20 & 4.49 & 3.86 & 4.41 & 5.35 & 5.97 & 4.35 & 5.72 & 6.24 & 3.11 \\
\hline $\mathrm{CaO}$ & 9.70 & 10.09 & 11.69 & 9.90 & 8.82 & 8.90 & 9.31 & 10.82 & 8.94 & 9.16 & 10.09 & 8.64 \\
\hline
\end{tabular}


Table 3 continued

\begin{tabular}{|c|c|c|c|c|c|c|c|c|c|c|c|c|}
\hline \multirow{2}{*}{$\begin{array}{l}\text { Sample } \\
\text { ID }\end{array}$} & \multicolumn{6}{|c|}{ KT95-9 D13 } & \multirow{2}{*}{$\begin{array}{l}\text { D14 } \\
\text { D14-05 }\end{array}$} & \multicolumn{5}{|l|}{ D16 } \\
\hline & D13-04 & D13-05 & D13-14 & D13-24 & D13-25 & D13-112 & & D16-101 & D16-103 & D16-105 & D16-109 & D16-202 \\
\hline $\mathrm{Na}_{2} \mathrm{O}$ & 2.91 & 2.63 & 2.56 & 3.20 & 3.14 & 3.00 & 2.45 & 2.20 & 2.42 & 2.80 & 2.23 & 3.15 \\
\hline $\mathrm{K}_{2} \mathrm{O}$ & 0.40 & 0.42 & 0.47 & 0.70 & 0.52 & 0.45 & 0.55 & 0.47 & 0.83 & 0.81 & 0.59 & 1.02 \\
\hline $\mathrm{P}_{2} \mathrm{O}_{5}$ & 0.14 & 0.08 & 0.47 & 0.64 & 0.13 & 0.08 & 0.08 & 0.10 & 0.07 & 0.09 & 0.07 & 0.10 \\
\hline Total & 99.86 & 99.79 & 99.63 & 99.66 & 99.71 & 99.84 & 99.32 & 99.77 & 99.35 & 99.99 & 100.19 & 99.98 \\
\hline $\mathrm{H}_{2} \mathrm{O}-$ & 0.75 & 1.04 & 1.45 & 0.78 & 0.64 & 0.68 & 0.41 & 0.65 & 0.33 & 0.19 & 0.63 & 0.31 \\
\hline LOI & 0.56 & 0.50 & 0.46 & 0.33 & 0.30 & 0.25 & 0.90 & 0.53 & 0.31 & 0.41 & 0.47 & 0.22 \\
\hline \multicolumn{13}{|c|}{ XRF analyzed trace elements (ppm) } \\
\hline Co & 26.8 & 30.7 & 37.5 & 33.4 & 21.4 & 32.0 & 17.6 & 25.6 & 16.4 & 31.8 & 33.4 & 14.7 \\
\hline $\mathrm{Cr}$ & 25.7 & 48.0 & 18.7 & 87.0 & 21.8 & 18.7 & 34.1 & 141 & 24.2 & 163 & 134 & 39.9 \\
\hline $\mathrm{Ba}$ & 25.6 & 29.0 & 42.1 & 50.6 & 36.8 & 36.3 & 53.3 & 64.8 & 75.2 & 78.9 & 61.3 & 76.4 \\
\hline $\mathrm{Nb}$ & 0.6 & 0.4 & 0.4 & 0.3 & 0.7 & 0.6 & 0.8 & 0.5 & 0.7 & 0.9 & 0.6 & 1.1 \\
\hline $\mathrm{Ni}$ & 14.0 & 17.3 & 19.8 & 30.4 & 8.8 & 12.2 & 27.3 & 40.9 & 19.3 & 43.6 & 43.3 & 18.4 \\
\hline $\mathrm{Pb}$ & 0.0 & nd & nd & nd & 0.1 & 0.4 & 0.4 & nd & 0.5 & 0.5 & nd & 0.4 \\
\hline $\mathrm{Rb}$ & 3.3 & 4.5 & 5.0 & 6.6 & 6.1 & 5.8 & 6.5 & 5.8 & 10.3 & 10.2 & 7.6 & 10.4 \\
\hline $\mathrm{Sr}$ & 194 & 181 & 198 & 226 & 199 & 186 & 244 & 275 & 258 & 231 & 269 & 253 \\
\hline Th & nd & 0.6 & 0.2 & 0.1 & 0.2 & 0.1 & 0.2 & 0.4 & 0.2 & 0.5 & nd & 0.6 \\
\hline $\mathrm{Y}$ & 26.4 & 22.0 & 24.6 & 39.5 & 25.0 & 22.0 & 15.3 & 15.4 & 13.1 & 17.2 & 10.6 & 15.5 \\
\hline $\mathrm{Zr}$ & 52.9 & 43.0 & 29.6 & 50.9 & 56.9 & 54.9 & 54.3 & 33.1 & 41.0 & 51.8 & 34.3 & 52.4 \\
\hline \multicolumn{13}{|c|}{ ICP-MS analyzed trace elements (ppm) } \\
\hline $\mathrm{Li}$ & 8.43 & 8.00 & 13.5 & 11.9 & 7.38 & 9.01 & 8.32 & 9.66 & 6.53 & 7.63 & 6.47 & 9.50 \\
\hline $\mathrm{Be}$ & 0.35 & 0.34 & 0.29 & 0.44 & 0.36 & 0.40 & 0.29 & 0.30 & 0.42 & 0.45 & 0.34 & 0.49 \\
\hline $\mathrm{Rb}$ & 2.58 & 4.16 & 4.88 & 5.70 & 5.23 & 5.09 & 5.99 & 4.92 & 8.84 & 9.08 & 6.22 & 9.12 \\
\hline $\mathrm{Sr}$ & 217 & 202 & 226 & 252 & 222 & 204 & 258 & 293 & 275 & 241 & 290 & 270 \\
\hline $\mathrm{Y}$ & 26.1 & 21.7 & 25.8 & 40.7 & 24.9 & 22.5 & 14.9 & 14.8 & 12.0 & 16.5 & 10.4 & 15.6 \\
\hline $\mathrm{Zr}$ & 46.4 & 38.9 & 28.4 & 44.7 & 50.5 & 50.7 & 49.3 & 29.3 & 34.1 & 46.5 & 29.9 & 45.8 \\
\hline $\mathrm{Nb}$ & 0.97 & 0.72 & 0.50 & 0.76 & 0.93 & 0.89 & 1.25 & 0.96 & 1.14 & 1.38 & 0.99 & 1.53 \\
\hline Mo & 0.54 & 0.45 & 0.38 & 0.54 & 0.56 & 0.55 & 0.85 & 0.48 & 0.69 & 0.74 & 0.56 & 0.59 \\
\hline $\mathrm{Sn}$ & 0.58 & 0.50 & 0.40 & 0.46 & 0.60 & 0.56 & 0.46 & 0.28 & 0.40 & 0.40 & 0.28 & 0.36 \\
\hline $\mathrm{Sb}$ & 0.12 & 0.06 & 0.19 & 0.21 & 0.08 & 0.06 & 0.80 & 0.10 & 0.11 & 0.09 & 0.28 & 0.06 \\
\hline Cs & 0.10 & 0.14 & 0.10 & 0.14 & 0.16 & 0.16 & 0.22 & 0.17 & 0.41 & 0.35 & 0.24 & 0.13 \\
\hline $\mathrm{Ba}$ & 34.6 & 32.2 & 30.1 & 45.2 & 40.7 & 43.8 & 54.3 & 58.0 & 74.1 & 75.8 & 56.6 & 87.3 \\
\hline $\mathrm{La}$ & 2.32 & 1.89 & 2.05 & 8.00 & 2.38 & 2.25 & 3.09 & 3.45 & 2.64 & 3.42 & 2.54 & 3.74 \\
\hline $\mathrm{Ce}$ & 6.36 & 5.08 & 4.33 & 15.5 & 6.78 & 6.40 & 7.51 & 7.27 & 5.97 & 8.04 & 5.84 & 8.43 \\
\hline $\operatorname{Pr}$ & 1.20 & 0.99 & 0.84 & 1.97 & 1.26 & 1.14 & 1.18 & 1.16 & 0.98 & 1.27 & 0.92 & 1.33 \\
\hline $\mathrm{Nd}$ & 6.65 & 5.61 & 4.65 & 10.3 & 6.84 & 6.21 & 5.75 & 5.64 & 4.83 & 6.26 & 4.46 & 6.33 \\
\hline $\mathrm{Sm}$ & 2.32 & 2.05 & 1.68 & 3.04 & 2.45 & 2.25 & 1.69 & 1.63 & 1.43 & 1.88 & 1.31 & 1.86 \\
\hline $\mathrm{Eu}$ & 0.88 & 0.77 & 0.66 & 1.08 & 0.92 & 0.86 & 0.66 & 0.59 & 0.55 & 0.67 & 0.51 & 0.66 \\
\hline $\mathrm{Gd}$ & 2.99 & 2.47 & 2.23 & 4.02 & 3.05 & 2.74 & 1.95 & 1.94 & 1.50 & 2.15 & 1.49 & 2.14 \\
\hline $\mathrm{Tb}$ & 0.62 & 0.52 & 0.47 & 0.78 & 0.65 & 0.59 & 0.39 & 0.37 & 0.32 & 0.42 & 0.29 & 0.41 \\
\hline Dy & 4.22 & 3.57 & 3.39 & 5.24 & 4.30 & 3.89 & 2.59 & 2.46 & 2.09 & 2.86 & 1.91 & 2.62 \\
\hline Ho & 0.94 & 0.79 & 0.79 & 1.18 & 0.94 & 0.86 & 0.56 & 0.54 & 0.46 & 0.60 & 0.40 & 0.56 \\
\hline $\mathrm{Er}$ & 2.62 & 2.17 & 2.30 & 3.31 & 2.66 & 2.39 & 1.57 & 1.51 & 1.29 & 1.73 & 1.12 & 1.56 \\
\hline $\mathrm{Tm}$ & 0.41 & 0.35 & 0.38 & 0.51 & 0.42 & 0.38 & 0.25 & 0.24 & 0.20 & 0.27 & 0.18 & 0.25 \\
\hline $\mathrm{Yb}$ & 2.76 & 2.26 & 2.56 & 3.34 & 2.80 & 2.53 & 1.74 & 1.61 & 1.42 & 1.84 & 1.23 & 1.63 \\
\hline $\mathrm{Lu}$ & 0.44 & 0.35 & 0.41 & 0.54 & 0.43 & 0.39 & 0.27 & 0.26 & 0.22 & 0.29 & 0.19 & 0.25 \\
\hline $\mathrm{Hf}$ & 1.58 & 1.28 & 0.97 & 1.50 & 1.66 & 1.59 & 1.47 & 0.95 & 1.10 & 1.33 & 0.94 & 1.40 \\
\hline $\mathrm{Ta}$ & 0.06 & 0.06 & 0.04 & 0.06 & 0.07 & 0.07 & 0.10 & 0.06 & 0.07 & 0.08 & 0.06 & 0.10 \\
\hline
\end{tabular}


Table 3 continued

\begin{tabular}{|c|c|c|c|c|c|c|c|c|c|c|c|c|}
\hline \multirow{2}{*}{$\begin{array}{l}\text { Sample } \\
\text { ID }\end{array}$} & \multicolumn{6}{|c|}{ KT95-9 D13 } & \multirow{2}{*}{$\begin{array}{l}\text { D14 } \\
\text { D14-05 }\end{array}$} & \multicolumn{5}{|l|}{ D16 } \\
\hline & D13-04 & D13-05 & D13-14 & D13-24 & D13-25 & D13-112 & & D16-101 & D16-103 & D16-105 & D16-109 & D16-202 \\
\hline $\mathrm{Tl}$ & 0.01 & 0.02 & 0.01 & 0.01 & 0.06 & 0.02 & 0.05 & 0.03 & 0.05 & 0.05 & 0.09 & 0.03 \\
\hline $\mathrm{Pb}$ & 6.84 & 8.48 & 4.02 & 12.3 & 4.43 & 3.28 & 8.59 & 13.0 & 2.23 & 3.09 & 2.50 & 4.18 \\
\hline Th & 0.14 & 0.11 & 0.09 & 0.19 & 0.17 & 0.16 & 0.28 & 0.26 & 0.27 & 0.36 & 0.26 & 0.33 \\
\hline $\mathrm{U}$ & 0.17 & 0.09 & 0.44 & 0.42 & 0.15 & 0.11 & 0.14 & 1.03 & 0.18 & 0.19 & 0.33 & 0.20 \\
\hline $\begin{array}{l}\mathrm{K}-\mathrm{Ar} \\
\text { dating }\end{array}$ & & $39.4 \pm 2.0 \mathrm{Ma}$ & & & & & & $45.0 \pm 1.8 \mathrm{Ma}$ & & & & \\
\hline
\end{tabular}

